\title{
In vitro Bioactivity Assessment of Novel Composites Based on Calcium Aluminate Cement
}

\author{
Cortés-Hernández DA ${ }^{1 *}$, Acuña-Gutiérrez $\mathrm{IO}^{1}$, Escobedo-Bocardo $\mathrm{JC}^{1}$, Almanza-Robles JMํㅜ ${ }^{1}$ García-Álvarez G ${ }^{1}$ and \\ Sánchez-Escobedo BA $^{1}$ \\ ${ }^{1}$ CINVESTAV-IPN Unidad Saltillo, Mexico
}

*Corresponding author: Cortés-Hernández DA, México-25900, Mexico

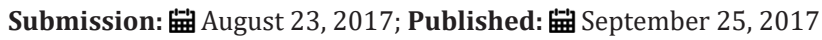

\begin{abstract}
The effect of pseudowollastonite $(\mathrm{Pw})$ additions on the in vitro bioactivity and compressive strength of composites based on calcium aluminate cement (CAC) was studied. Composites were prepared by mixing calcium aluminate clinker with pseudowollastonite (5 and $10 \mathrm{wt} \%)$ at a w/c ratio of $0.4(0.015 \mathrm{M} \mathrm{LiCl}$ aqueous solution). In order to assess the in vitro bioactivity and compressive strength, composites (CAC 5Pw and CAC 10Pw) were immersed in simulated body fluid (SBF) at $37^{\circ} \mathrm{C}$ for $1,7,14$ or 21 days. Analyses of the surface cements after immersion in SBF revealed the formation of a bonelike apatite layer in both composites, increasing the amount of this $\mathrm{Ca}$, $\mathrm{P}$ rich compound as the $\mathrm{Pw}$ content was increased. The compressive strength of the composites decreased as the pseudowollastonite content was increased. However, the strength of the composites was higher than that established for bone cements in the ASTM F451 standard.
\end{abstract}

Keywords: Calcium aluminate; Bioactive cements; Pseudowollastonite

\section{Introduction}

Nowadays, there are many research works directed towards the development of bioglasses ceramics (calcium phosphates $[1,2]$, bio glasses $[3,4]$, etc.). However, some specific applications require the in situ conformation of the materials, fact that limits their applications. An interesting research field is that of bioactive cements, due to the ability of these cements to be molded in situ. Many studies have been focused on obtaining calcium phosphate cements $[5,6]$ since they have a composition similar to that of human bone. However, due to the low mechanical properties of these materials, their application has been limited to low strength areas. Calcium aluminate cement has been of great interest to researchers as bone cement [7] due to their high mechanical properties [8] and bioactivity $[9,10]$. However, this type of cement shows long setting times, which limits their use for some medical applications. In previous studies [11] it was determined that the addition of $\mathrm{LiCl}$ to the CAC in order to decrease the setting time inhibited the bioactivity of this cement. For this reason, the effect of the addition of pseudowollastonite on bioactivity and compressive strength of a CAC exposed to SBF was studied in this paper.

Experimental

\section{Materials}

Calcium aluminate clinker was obtained by solid state reaction from a mixture of reagent grade chemicals of calcium carbonate $\left(\mathrm{CaCO}_{3}\right.$, Aldrich $99 \%$ purity) and alumina $\left(\mathrm{Al}_{2} \mathrm{O}_{3}\right.$, Aldrich
$99.5 \%$ purity). The mixture was heat treated at $1450{ }^{\circ} \mathrm{C}$ for $4 \mathrm{~h}$. Pseudowollastonite was synthesized by sol-gel using tetraethyl orthosilicate (TEOS, Aldrich 98\% purity) and calcium nitrate (Aldrich 99\% purity) as precursors. Both materials obtained were milled until reaching an average particle size of $22 \mu \mathrm{m}$.

\section{Sample preparation}

The samples were prepared by mixing calcium aluminate clinker with the corresponding amount of pseudowollastonite (5 or $10 \%)$ at a w/c ratio of $0.4(0.015 \mathrm{M} \mathrm{LiCl}$ aqueous solution) until a paste was formed. Pastes were casted into two different nylamid molds to obtain samples for the in vitro bioactivity and compressive strength testing assays. All samples were set for $1 \mathrm{~h}$. The composites were identified as CAC 5Pw (5\% pseudowollastonite) and CAC $10 \mathrm{Pw}$ (10\% pseudowollastonite).

\section{In vitro bioactivity assessment}

The simulated body fluid (SBF) used in these tests was prepared following the procedure described by Kokubo et al. [12]. After setting, each sample was immersed in $200 \mathrm{ml}$ of SBF and placed into an incubator at $37{ }^{\circ} \mathrm{C}$ for 21 days. After immersion, samples were stored in desiccators. The surface of the composite samples was analyzed before and after immersion in SBF by scanning electron microscopy (SEM; Philips, XL30 ESEM), energy dispersive spectroscopy (EDS; EDAX, Pegasus) and X-ray diffraction (XRD; Philips, Xpert). 


\section{Compressive strength}

Before compressive strength evaluation, samples were immersed in SBF for $1,7,14$ or 21 days at $37^{\circ} \mathrm{C}$. The compressive load was applied at a crosshead speed of $0.5 \mathrm{~mm} / \mathrm{min}$. The mechanical strength evaluation was performed according to the ASTM F 451[13].

\section{Setting times}

The initial and final setting times of cements were evaluated using a Vicat needle (ASTM C 191) [14].

\section{Results and Discussion}

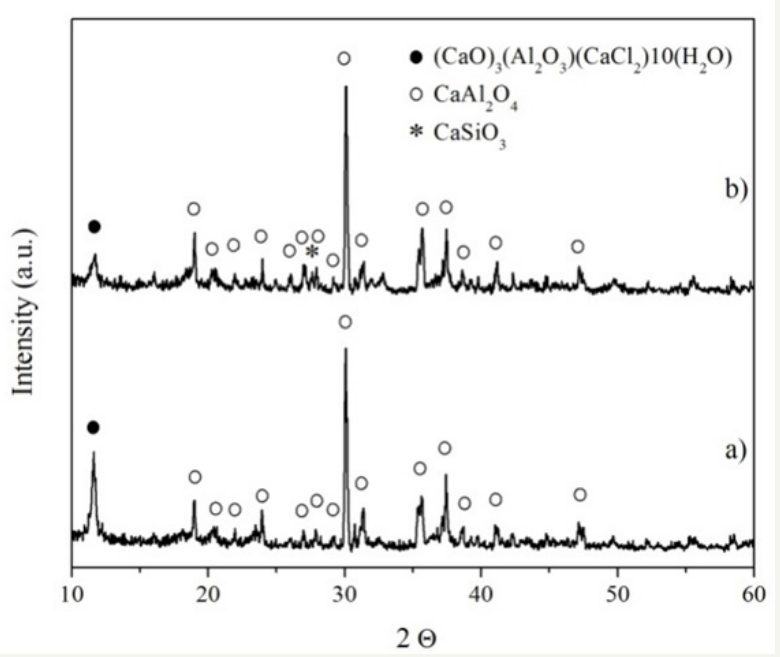

Figure 1: XRD patterns of composite surfaces before immersion in SBF: 1a: CAC 5Pw; 1b: CAC 10Pw.

Figure 1 shows the XRD patterns of the surface composites (CAC 5Pw and CAC 10Pw) before immersion in SBF. Peaks corresponding tonon-hydratedcalcium aluminate [JCPDS 070-0134; $\mathrm{CaAl}_{2} \mathrm{O}_{4}$ ] and Friedel's salt [JCPDS 089-52-94; $(\mathrm{CaO})_{3}\left(\mathrm{Al}_{2} \mathrm{O}_{3}\right)\left(\mathrm{CaCl}_{2}\right)\left(\mathrm{H}_{2} \mathrm{O}\right)_{10}$ ] wereobserved in both patterns (Figure $1 \mathrm{a} \& 1 \mathrm{~b}$ ). It can be observed that the intensity of the peaks corresponding to Friedel's salt decreased as the quantity of pseudowollastonite increased, this fact is possibly due to a decrease in porosity of the composite by the addition of pseudowollastonite. This salt is formed by the reaction of CAC with the chloride ions of the aqueous solution [15]. In the XRD pattern corresponding of the CAC 10Pw (Figure 1b), also pseudowollastonite [JCPDS 074-08-74; $\mathrm{CaSiO}_{3}$ ] was detected. No peaks corresponding to pseudowollastonite were observed on the pattern of CAC 5Pw (Figure 1a) due to the sensibility of this technique.

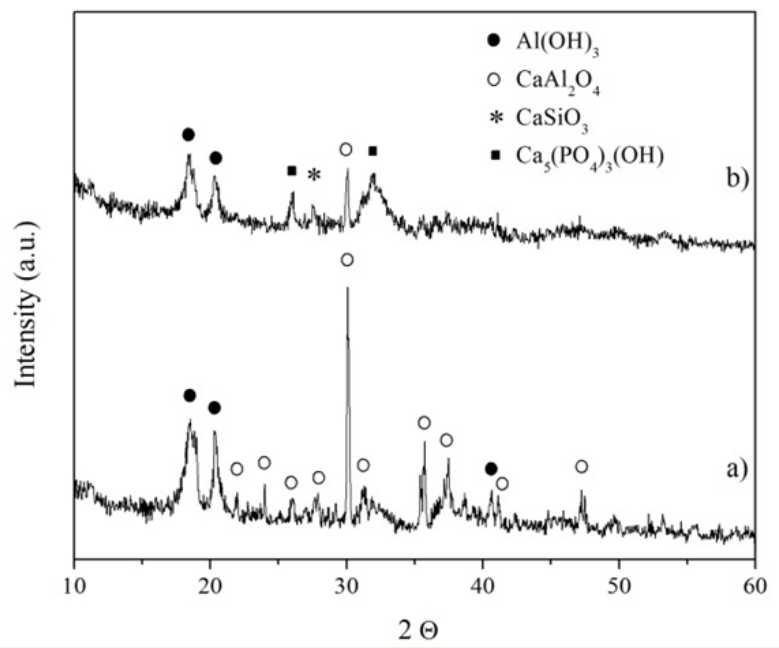

Figure 2: XRD patterns of composite surfaces after2 1 days of immersion in SBF: 2a: CAC 5Pw; 2b: CAC 10Pw.

Figure 2 shows the XRD patterns of the surface composites (CAC 5Pw and CAC 10Pw) after 21 days of immersion in SBF. Peaks corresponding tonon-hydrated calcium aluminate [JCPDS 0700134; $\mathrm{CaAl}_{2} \mathrm{O}_{4}$ ], aluminum hydroxide [JCPDS 074-1775; $\mathrm{Al}(\mathrm{OH})_{3}$ ] and pseudowollastonite[JCPDS 074-08-74; $\mathrm{CaSiO}_{3}$ ] were observed in both patterns. Peaks corresponding to HA be observed in the pattern corresponding to CAC 10Pw (Figure 2b). This compound is formed in bioactive materials when they are exposed to SBF. As observed, the increase in the amount of pseudowollastonite in the composite increased the bioactivity of the material.
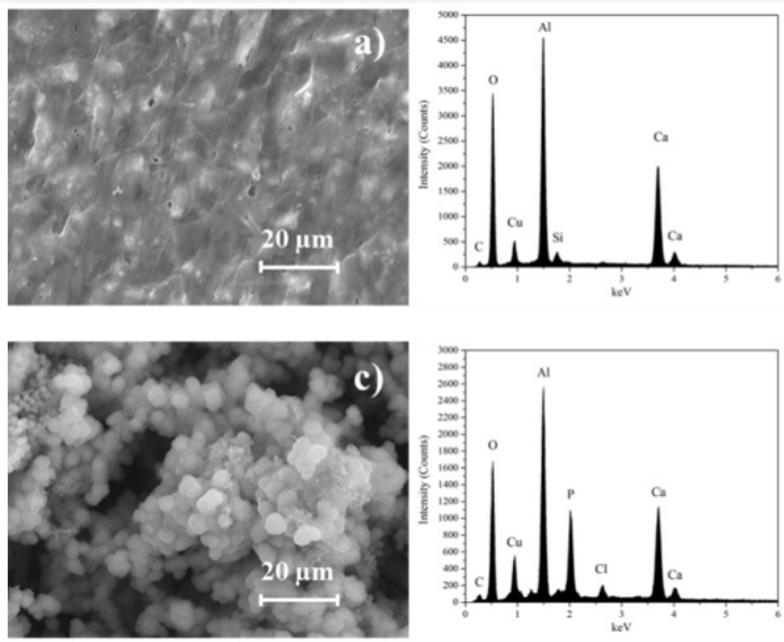
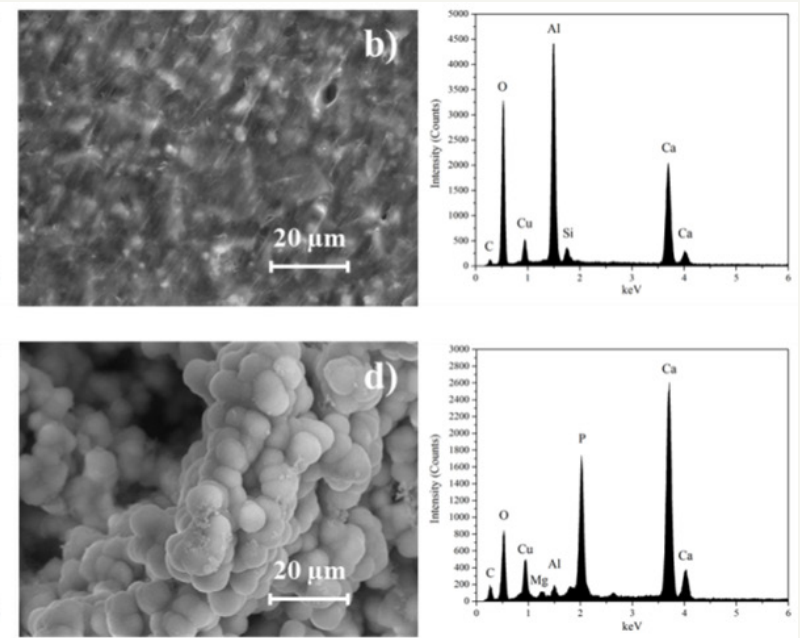

Figure 3: SEM images and EDS spectra of the surface composites before immersion in SBF: 3a: CAC 5 Pw; 3b: CAC 10 Pw; and after 21 days immersion in SBF: 3c: CAC 5Pw; 3d: CAC $10 \mathrm{Pw}$. 
Figure 3 shows SEM images and EDS spectra of the composites (CAC 5Pw and CAC 10Pw) before and after 21 days of immersion in SBF. Before immersion (Figure 3a \& 3b), a rough surface was observed on both composites. In the corresponding EDS spectra, the presence of $\mathrm{Al}, \mathrm{Ca}, \mathrm{Si}$ and $\mathrm{O}$, main elements of calcium aluminate and pseudowollastonite was detected, which was corroborated by XRD (Figure 1). Figures 3c \& 3d correspond to the composites CAC $5 \mathrm{Pw}$ and CAC $10 \mathrm{Pw}$ after 21 days of immersion in SBF. It can be observed in both SEM images that a thick and homogenous ceramic layer, constituted by $\mathrm{Ca}$, P-rich agglomerates, fact corroborated for the corresponding EDS spectra, was formed in the surfacecements. The Ca, P-rich layer was identified as HA by XRD (Figure 2). The bioactivity of the cements increased as the pseudowollastonite amount was increased.

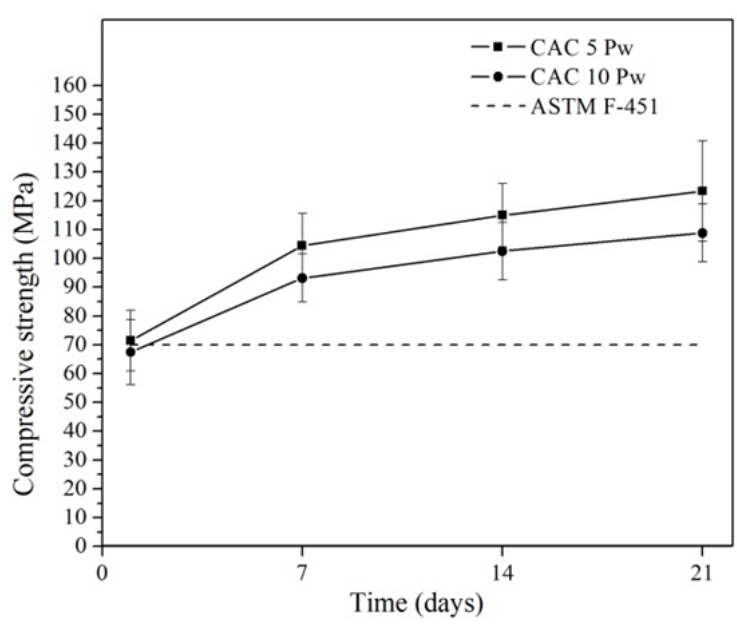

Figure 4: Compressive strength of composites as a function of immersion time in SBF.

Figure 4 shows the compressive strength of the composites as a function of immersion time in SBF. As observed, the compressive strength decreased as the pseudowollastonite content was increased. The initial values of strength (1 day of immersion in $\mathrm{SBF}$ ) of cements were around 70MPa. After 21 days of immersion in SBF composites (CAC 5Pw and CAC 10Pw) shows appropriates mechanical properties. According to the ASTM F-451, a minimum value of $70 \mathrm{MPa}$ is required for bone cements.

When comparing setting time of both cements, no significant changes were observed in the initial (23 and 20min) and final (2.5 and $2.3 \mathrm{~min}$ ) setting times obtained for CAC 5Pw and CAC $10 \mathrm{Pw}$, respectively.

\section{Conclusion}

Novel composites based in calcium aluminate cement were obtained. After 21 days of immersion in SBF a homogeneous and thick apatite layer was observed on the surface cements (CAC 5Pw and CAC $10 \mathrm{Pw}$ ). A higher bioactivity was observed on cements with content of $10 \%$ of pseudowollastonite. The cements showed appropriate compressive strength. The results obtained indicate that cements can be highly potential bone cements.

\section{Acknowledgement}

The authors gratefully acknowledge CONACYT, México for the scholarship 219442 granted to I.O. Acuña Gutiérrez.

\section{References}

1. Judith AJ, Serena MB, Antony DA, William B (2008) Biological control of apatite growth in simulated body fluid and human blood serum. Journal of Materials Science: Material in Medicine 19(4): 1823-1829.

2. Iain RG, William B (2002) Novel synthesis and characterization of an ABtype carbonate-substituted hydroxyapatite. J Biomed Mater Res 59(4): 697-708.

3. Ravarian R, Moztarzadeh F, Solati Hashjin M, Rabiee SM, Khoshakhlagh P, et al. (2010) Synthesis, characterization and bioactivity investigation of bioglass/hydroxyapatite composite. Ceramics International 36(1): 291297.

4. Anbalagan B, Gerard B, Sanjeevi K, Jean M, Avito R, et al. (2007) Development and in vitro characterization of sol-gel derived $\mathrm{CaO}-\mathrm{P}_{2} \mathrm{O}_{5}$ $\mathrm{SiO}_{2}$-ZnO bioglass. Acta Biomater 3(2): 255-262.

5. Young BK, Byung ML, Myung CL, Insup N, Sung-Jae L, et al. (2013) Preparation and Characterization of Calcium Phosphate Cement of $\alpha$-Tricalcium Phosphate-Tetracalcium Phosphate-Dicalcium Phosphate System Incorporated with Poly $(\gamma$-glutamic acid). Macromolecular Research 21(8): 892-898.

6. Jingtao Z, Weizhen L, Verena S, Franck T, Jean-Michel B (2014) Calcium phosphate cements for bone substitution: Chemistry, handling and mechanical properties. Acta Biomater 10(3): 1035-1049.

7. Larissa MS, De Castro, Ivone RDO, Lucas NT, Adalberto LR, et al. (2011) Effects of a novel Calcium Aluminate Cement on the Early Events of the Progression of Osteogenic Cell Cultures. Brazilian Dental Journal 22(2): 99-104.

8. Oliveira IR, Pandolfelli VC, Jacobovitz M (2010) Chemical, physical and mechanical properties of a novel calcium aluminate endodontic cement. International Endodontic Journal 43(12): 1069-1076.

9. Jesper L, Fredrik S, Tobias J, Hakan E, Cornelis HP (2008) A comparative study of the bioactivity of three material for dental applications. Dental Materials 24(5): 653-659.

10. Tan YN, Liu Y, Zhang ZB, Huang BY, Grover LM (2011) Hydroxyapatite formation on surface of calcium aluminate cements. Advances in applied ceramics 110(8): 464-468.

11. Acuña-Gutiérrez IO, Escobedo-Bocardo JC, Almanza-Robles JM, CortésHernández DA, Saldívar-Ramírez MMG, et al. (2017) Development of LiClcontaining calcium aluminate cement for bone repair and remodeling applications. Mater Sci Eng C Mater Biol Appl 70(Pt 1): 357-363.

12. Tadashi K, Hiroaki T (2006) How useful is SBF in predicting in vivo bone bioactivity? Biomaterials 27(15): 2907-2915.

13.1999, Standard Specification for Acrylic Bone Cement, ASTM F 451, West Conshohocken, PA, USA.

14. 2004, Standard Test Method for Time of Setting of Hydraulic Cement by Vicat Needle, ASTM C 191, West Conshohocken, PA, USA.

15. San Juán MA (1997) Formation of chloroaluminates in calcium aluminate cements cured at high temperatures and exposed to chloride solutions. J Mater Sci 32(23): 6207-6213. 